\title{
A regional anesthesia-based "swing" operating room model reduces non-operative time in a mixed orthopedic inpatient/ outpatient population
}

\section{Un modèle de salle « en rotation » basée sur l'anesthésie régionale en orthopédie réduit le temps non-opératoire dans une population mixte de patients ambulatoires et hospitalisés}

\author{
Paul Mercereau, MD • Bobby Lee, MD • \\ Stephen J. Head, MD • Stephan K. W. Schwarz, MD, PhD
}

Received: 26 April 2012/Accepted: 16 July 2012/Published online: 31 July 2012

(C) Canadian Anesthesiologists' Society 2012

\begin{abstract}
Purpose We recently reported on the efficacy of a new "swing" room model involving two alternating ORs and regional anesthesia in increasing operating room (OR) throughput in a dedicated ambulatory orthopedic surgery facility. The purpose of this study was to evaluate this model in a main OR suite setting with typical mixed inpatient/outpatient cases.

Methods We conducted a retrospective matched-pair cohort study of 133 upper extremity surgery patients treated in the swing room model under ultrasound-guided brachial plexus blockade. We compared this cohort with case-matched historical controls treated in the traditional single OR model under general anesthesia. The primary endpoint was non-operative time, defined as the interval between skin closure and incision in the following case.
\end{abstract}

Author contributions Paul Mercereau performed the data abstraction, was involved in the statistical analysis, and wrote the first draft of the manuscript. Bobby Lee and Stephan K.W. Schwarz were involved in data interpretation. Bobby Lee, Stephen J. Head, and Stephan K.W. Schwarz were involved in the concept and design of the study. Bobby Lee and Stephen J. Head were involved in manuscript editing. Stephan K.W. Schwarz was involved in data analysis, and he critically revised the manuscript.

P. Mercereau, MD - B. Lee, MD · S. J. Head, MD .

S. K. W. Schwarz, MD, PhD

Department of Anesthesiology, Pharmacology \& Therapeutics,

The University of British Columbia, Vancouver, BC, Canada

P. Mercereau, MD - B. Lee, MD · S. J. Head, MD .

S. K. W. Schwarz, MD, PhD ( $)$

Department of Anesthesia, St. Paul's Hospital, Providence Level

3, 1081 Burrard Street, Vancouver, BC V6Z 1Y6, Canada

e-mail: stephan.schwarz@ubc.ca
Secondary endpoints included throughput estimated as the median number of cases per eight-hour day, postanesthesia care unit (PACU) bypass rates, and postoperative pain/ nause and vomiting (PONV) intervention rates.

Results Compared with the control group, non-operative times in the swing room group were faster (swing: median $19 \mathrm{~min}$; interquartile range [IQR 8-31] vs control: median 57 min; IQR [49-65]; $P<0.0001)$. In the swing room model, the estimated daily throughput was $33 \%$ greater (swing: median 5.6 cases; IQR [5.0-6.2] vs control: median 4.2 cases; IQR [4.0-4.4]; $P<0.0001)$, and the PACU bypass rate was higher (swing: $60 \%$ vs control: $0 \% ; P<0.0001$ ). Fewer patients received postoperative opioids (swing: $20 \%$ vs control: $82 \% ; P<0.0001$ ) and treatment for PONV (swing: $2 \%$ vs control: $20 \% ; P<0.0001)$ in the swing room model. Conclusion The implementation of a "swing" room care model based on ultrasound-guided regional anesthesia in a typical mixed inpatient/outpatient population decreased non-operative times, increased throughput, and improved recovery profiles compared with case-matched historical controls in the traditional model under general anesthesia.

\footnotetext{
Résumé

Objectif Nous avons récemment parlé de l'efficacité pour l'augmentation du nombre de cas traités d'un nouveau modèle de salle "en rotation » impliquant l'utilisation en alternance de deux salles d'opération et l'utilisation de l'anesthésie régionale dans un centre spécialisé en chirurgie orthopédique ambulatoire. L'objectif de cette étude était d'évaluer ce modèle dans le cadre d'un ensemble de salles d'opérations accueillant aussi bien des patients hospitalisés que des patients ambulatoires.
} 
Méthodes Nous avons mené une étude rétrospective sur une cohorte de 133 patients appariés par paires qui devaient subir une chirurgie du membre supérieur avec bloc du plexus brachial sous guidage échographique dans le modèle de salle "en rotation». Nous avons comparé cette cohorte à des cas contrôles historiques appariés et traités dans une salle d'opération traditionnelle unique sous anesthésie générale. Le critère d'évaluation principal était le temps non-opératoire, défini comme étant le délai écoulé entre la fermeture de la peau d'un patient et l'incision sur le patient suivant. Les critères d'évaluation secondaires ont inclus la capacité estimée, c'est-à-dire le nombre médian de cas par période de huit jours, les taux d'évitement de la salle de réveil et les taux d'interventions pour douleurs/nausées et vomissements postopératoires.

Résultats Comparativement au groupe contrôle, les temps non-opératoires dans le groupe "salle en rotation » ont été plus courts (durée médiane [plage interquartile] : 19 [8-31] contre 57 [49-65] minutes; $P<0,0001)$, capacitéjournalière estimée supérieure de $33 \%$ (nombre de cas : 5,6 [5,0-6,2] contre 4,2 [4,0-4,4]; $P<0,0001)$ et le taux d'évitement de la salle de réveil a été plus élevé $(60 \%$ contre $0 \%$; $P<0,0001)$. Moins de patients ont reçu des morphiniques postopératoires (20\% contre $82 \% ; P<0,0001)$ et un traitement pour douleur/nausées et vomissements postopératoires (2\% contre $20 \%$; P $<0,0001)$ dans le modele de salle " en rotation".

Conclusion La mise en auvre d'un modele de soins de « salle en rotation » basée sur une anesthésie régionale guidée par échographie pour une population mixte de patients hospitalisés et ambulatoires a diminué les temps non opératoires, augmenté la capacité et amélioré les profils de récupération.

Reduction of surgical wait lists continues to be an issue of priority in the Canadian health care system. ${ }^{1,2}$ In 2010, the Fraser Institute showed that the median wait in orthopedic surgery from referral to treatment was as long as 35.6 weeks. ${ }^{3}$ Surgeons cited the most frequent factor governing wait lists as a lack of available operating time. ${ }^{4}$ Consequently, there is a need to develop new perioperative care models that increase efficiency and throughput within the existing time allotment in the operating room (OR).

Anesthesia care can significantly enhance the perioperative process. For example, in upper extremity surgery, use of brachial plexus blockade (BPB) rather than general anesthesia (GA) leads to reduced postoperative pain and analgesic requirements, ${ }^{5-13}$ decreased postoperative nausea and vomiting (PONV), ${ }^{5-9,13}$ and reduced length of stay in the postanesthesia care unit (PACU) ${ }^{6-14}$ The recent introduction of ultrasound technology has led to an increased interest in regional anesthesia as its use is associated with numerous advantages, including the potential for accelerated onset times and greatly improved success rates. ${ }^{15-17}$ These attributes render ultrasound-guided regional anesthesia an attractive component of perioperative care models targeted to increase efficiency and throughput.

Another component, aimed at minimizing productivity losses due to turnover between cases, is parallel processing. ${ }^{18}$ We recently co-developed and implemented a novel care model for a dedicated ambulatory facility for extremity surgery. The model is based on ultrasoundguided regional anesthesia and parallel processing. ${ }^{13}$ In this model, care is provided in two operating rooms and an adjacent multipurpose unit that serves simultaneously as a preoperative admission area, an ultrasound-equipped regional anesthesia room, and a postoperative recovery area. This "swing" room model involves one single anesthesiologist with one anesthesia assistant, four nurses, a surgical assistant, and one single attending surgeon who "swings" between the ORs to operate on patients in whom regional anesthesia is present upon OR entry. We recently reported that this outpatient-based model is associated with a decreased turnover time and increased throughput compared with the traditional model of GA in a single OR. ${ }^{13}$ However, this model may not be readily reproducible in hospitals treating a mixed inpatient/outpatient case load that includes procedures of longer duration. In November 2008, we introduced a "swing" room model in the main OR suite. Compared with our dedicated ambulatory facility model, this model involves two anesthesiologists, one of whom is dedicated to a regional anesthesia area with the aid of one anesthesia assistant. The two designated "swing" ORs are collectively staffed by four nurses. Ultrasound-guided regional anesthesia is performed in the block area while the preceding case is underway. Upon surgical closure of a case, the subsequent patient is transferred from the block area to the adjacent vacant OR, and the surgeon "swings" over to that room while regional anesthesia is performed on the following patient.

Our primary objective was to test the hypothesis that the swing room model would result in reduced non-operative time $^{19}$ compared with the prior standard of care based on GA in a single OR. Our secondary objectives were to determine the effects of this model on case throughput, PACU bypass rate and length of stay, and postoperative interventions for pain and PONV.

\section{Methods}

Following institutional Research Ethics Board approval (St. Paul's Hospital, Providence Health Care, Vancouver, BC, Canada; April 22, 2011), we performed a retrospective 
matched-pair cohort study based on a chart review of all patients who underwent upper extremity surgery under the swing room model in the main OR suite from November 2008 to March 2010, which corresponded with the first 17 months of the model's implementation. Corresponding charts were identified from the hospital's surgical database and from the daily filed OR slates on which the swing room slates (booked in two adjacent ORs and assigned to the same orthopedic surgeon) were clearly labelled. These were different patients from those in the ambulatory cohort of Outpatient Department Surgical Procedure Room patients studied in our recent report, and they were also treated in a different facility. ${ }^{13}$ The Research Ethics Board waived the requirement for informed consent from the individual subjects. We matched swing room model cases with historical control cases completed from 2006 to 2010 under GA in the context of a conventional "single surgeon-single anesthesiologist-single OR" model as closely as possible (i.e., identical anatomical location and surgical procedure). If an exact match could not be identified, the minimum acceptable matching criteria were identical anatomic location (e.g., phalanx surgery matched to phalanx surgery) and bony $v s$ soft tissue surgery. If more than one match existed, the historical control with the closest surgical duration was used. The following exclusion criteria were applied: age $<18 \mathrm{yr}$, scheduled primary anesthetic technique other than BPB, and inability to identify a matching historical control. The abstracted data included patient demographics, surgeon, type of surgery, non-operative time (defined below), nursing interventions for PONV and/or postoperative pain, timing and morphine-equivalent dose of administered analgesics, PACU bypass rate, and PACU length of stay. We abstracted the postoperative opioids and rescue antiemetics administered in the surgical daycare centre (phase II recovery area) to patients who bypassed the PACU.

The primary outcome variable was non-operative time as defined by the time between skin closure on one case and skin incision on the following case. ${ }^{19}$ As a secondary outcome variable, we estimated case throughput, which we expressed as the median number of comparable cases possible in an eight-hour day (beginning at the time of surgical incision on the first patient of the day) and calculated using the formula below ${ }^{13}$ :

$N=1+\frac{480-S-C}{S+N O}$

where:

$N=$ number of cases possible in an eight-hour day;

1 because no turnover is associated with the first case of the day; $480=$ number of minutes in eight hours;

$S=$ mean surgical time;

$C=$ time from surgical closure until OR exit; and

$N O=$ non-operative time
Statistical analysis

Statistical analyses were conducted on an intention-to-treat basis using Prism ${ }^{\circledR}$ version 5 (GraphPad, San Diego, CA, USA), Microsoft ${ }^{\circledR}$ Excel $^{\circledR}$ version 2003 (Microsoft Corporation, Redmond, WA, USA), and SSPS ${ }^{\circledR}$ version 20 software (IBM, Armonk, NY, USA). For continuous variables, we used the D'Agostino \& Pearson omnibus test to assess normality, and we performed comparisons using the Mann-Whitney test; such results are expressed as median (interquartile range [IQR]). We approximated differences between medians by calculating the Hodges-Lehmann estimator and its 95\% confidence interval (CI), and we analyzed categorical data with the Fisher's exact test. Statistical significance was defined as $P<0.05$. To be conservative, analyses were two-tailed and based on group rather than on subject-to-subject comparisons.

\section{Results}

From November 2008 to March 2010, 207 eligible upper extremity procedures were performed in the swing room model, and 133 of these qualified for inclusion in the analysis. The 133 cases came from 39 "swing room days", and cases per day ranged in number from one to six as slates were not limited to upper extremity surgery; hence, they frequently included procedures that did not meet inclusion criteria. Four cases could not be matched. Details of the matching sequence can be seen in the study flow chart (Figure 1). In two patients, rescue GA was performed due to block failure, yielding a 98.5\% (131/133) block success rate.

Both groups were similar with respect to demographic data, surgical duration, and types of procedures (Table 1). Non-operative time cannot be calculated for the first case of the day, so the available sample size for this variable was less than 133 (swing room group, $n=104$; control group, $n=110$; Table 2). ${ }^{13}$ In the swing room group, the number of cases possible in an eight-hour day could not be calculated for two patients because the time from surgical closure to OR exit had not been recorded. Non-operative time was significantly shorter in the swing room group than in the historical control group (median $19 \mathrm{~min}$; IQR [8-31]; $n=104 v s$ median $57 \mathrm{~min}$; IQR [49-65]; $n=110$, respectively; $P<0.0001$ ). Calculation of the Hodges-Lehmann estimator showed a difference between medians of $38 \mathrm{~min}$ (95\% CI 34 to 42). Eleven per cent of the 104 swing room patients had a negative non-operative time compared with none of the patients in the control group, reflecting the ability of the attending surgeon to commence the next procedure while the surgical resident or fellow completed closure on the previous patient. The difference in nonoperative time was still significant $(P<0.0001)$ when all 
Study flow chart

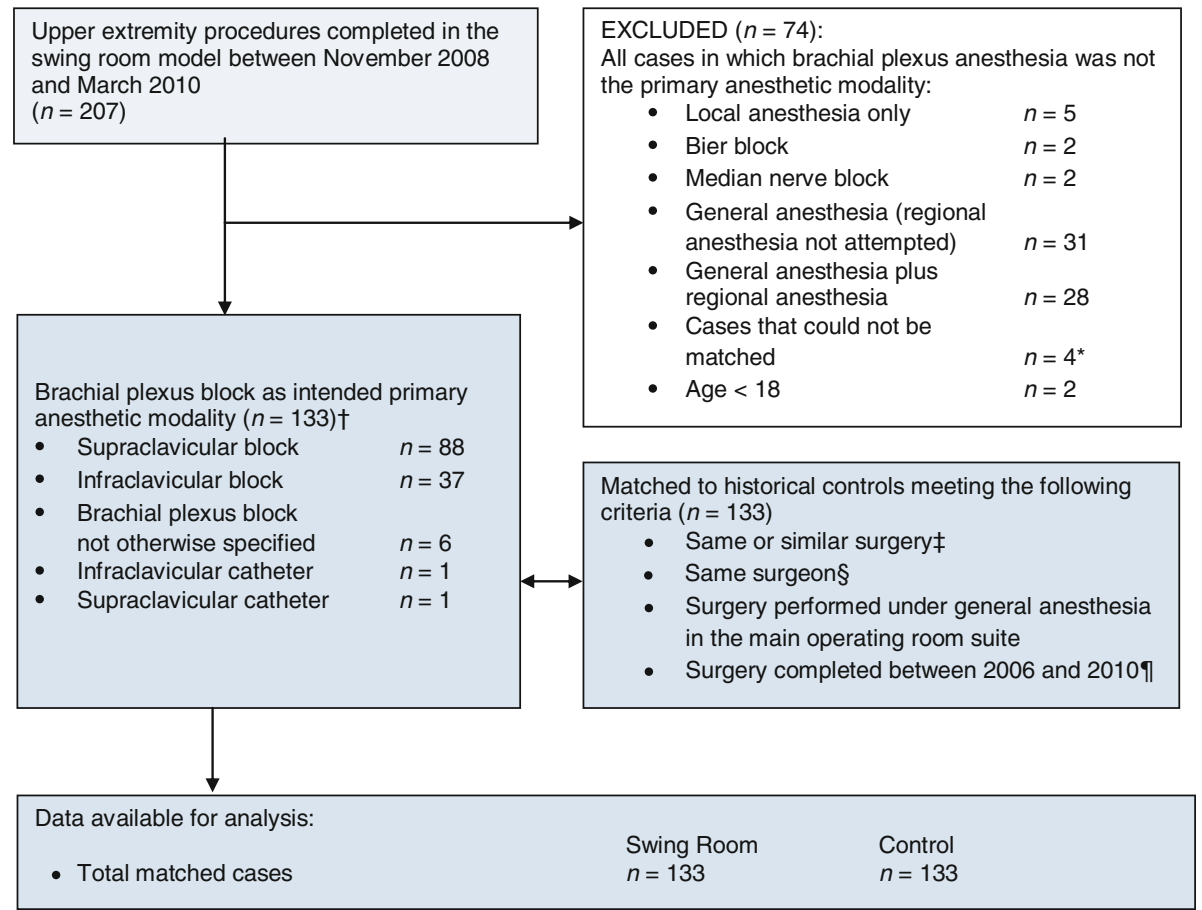

Figure 1 Study flow chart. *Four cases could not be matched as no suitable similar case performed in the absence of brachial plexus anesthesia could be identified using available controls from 2006-2010. †n two cases, single-shot brachial plexus block failed (one supraclavicular, one infraclavicular), necessitating the induction of general anesthesia. *An attempt was made to match surgical procedures as closely as possible (i.e., same anatomic location and

patients with negative values were either excluded from the analysis or assigned "zero" as non-operative time.

The implementation of the swing room model produced a $33 \%$ increase in estimated case throughput compared with the historical control group (conservatively based on a mean surgical duration per case of 70 min (median, 5.6 cases vs 4.2 cases per eight-hour day, respectively; $P<0.0001)$. Calculation of the Hodges-Lehmann estimator showed a difference between medians of 1.4 cases per eight-hour day (95\% CI 1.2 to 1.6) (Table 2).

All postoperative study endpoints were markedly improved in the swing room group, including the rates of PACU bypass, postoperative opioid administration, and treatment for PONV (Table 2).

\section{Discussion}

This study shows that the implementation of a swing room perioperative care model - based on ultrasound-guided regional anesthesia and parallel processing - to a typical mixed inpatient/outpatient population in the setting of a hospital's main OR suite was associated with significantly same operation). If exact matches were not available, the minimum acceptable matching criteria were same anatomic location and bony $v s$ soft tissue surgery. ${ }^{\S}$ In seven cases, the surgeon could not be matched; matching was relegated to another surgeon who had worked regularly in both settings. "If more than one match existed, the historical control with the closest surgical duration was used

decreased non-operative times and improved recovery profiles for patients undergoing upper extremity orthopedic surgery. Similar results were found in our previous work in the ambulatory setting. ${ }^{13}$ The present findings suggest that such a model can be generalized without the infrastructure investment necessary to support our outpatient swing-room facility and, thus, would be applicable to a wide range of other institutions.

The improved case throughput in the swing room group can be attributed to the concurrent execution of two levels of parallel processing. First, the use of a dedicated regional anesthesia block area facilitates early placement of BPB and allows sufficient time to ensure surgical anesthesia before the incision is performed, which likely contributed significantly to the high block success rate. When regional anesthesia with a dedicated block area is compared with GA, previous research has shown that regional anesthesia decreases anesthesia-controlled time (traditionally defined as the interval between surgical closure and OR exit of one patient plus the interval between the next patient entering the OR and being "anesthesia ready") for upper extremity surgery, ${ }^{12,14,20}$ although the time saved was small (from 4 to $11 \mathrm{~min})$. However, it is important to point out that 
Table 1 Patient demographic and surgical data

\begin{tabular}{lrr}
\hline & $\begin{array}{r}\text { Swing room } \\
(n=133)\end{array}$ & \multicolumn{1}{c}{$\begin{array}{c}\text { Control } \\
(n=133)\end{array}$} \\
\hline Male & $73(55 \%)$ & $66(50 \%)$ \\
Age (yr) & $48[37-60]$ & $52[35-63]$ \\
Body mass index $\left(\mathrm{kg} \cdot \mathrm{m}^{-2}\right)$ & $25[22-29]$ & $25[22-28]$ \\
ASA physical status & & \\
I or II & $108(81 \%)$ & $107(80 \%)$ \\
III or IV & $25(19 \%)$ & $26(20 \%)$ \\
Surgical duration (min) & $65[43-88]^{*}$ & $64[45-86]^{\dagger}$ \\
Ambulatory surgery & $118(89 \%)$ & $124(93 \%)$ \\
Type of surgery & & \\
Fracture fixation & $23(18 \%)$ & $26(20 \%)$ \\
Ligament/tendon/nerve & $23(18 \%)$ & $23(18 \%)$ \\
Arthroplasty & $20(15 \%)$ & $19(14 \%)$ \\
Tumour/mass excision/biopsy & $8(6 \%)$ & $8(6 \%)$ \\
Joint fusion/arthrodesis & $12(9 \%)$ & $13(10 \%)$ \\
Arthroscopy & $7(5 \%)$ & $8(6 \%)$ \\
Hardware removal & $9(7 \%)$ & $9(7 \%)$ \\
Osteotomy & $12(9 \%)$ & $8(6 \%)$ \\
Miscellaneous & $19(15 \%)$ & $19(15 \%)$ \\
\hline Data are expresse & &
\end{tabular}

Data are expressed as number (\%) or median [interquartile range]. * Mean, $70 \mathrm{~min} ;{ }^{\dagger}$ Mean, $69 \mathrm{~min} ;{ }^{*}$ comprises carpal tunnel release, debridement, hematoma drainage, carpectomy, bone grafting, joint reconstruction, bursa excision, synovectomy, elbow release, digit amputation, Dupuytren's release, and fasciotomy. ASA = American Society of Anesthesiologists

traditional definitions of "anesthesia-controlled time" have limited applicability in our model because surgical anesthesia is usually present when the patient enters and leaves the OR, and BPB is administered while the previous case is underway. Our findings that patients treated in the swing room model under regional anesthesia had faster OR entry to incision times than those receiving GA likely reflect this fact. In addition, in the swing room group, we found a small but highly significant reduction in the time from skin closure to OR exit, presumably due to the absence of the requirement for emergence from general anesthesia. These data indicate that the regional anesthesia component of the swing room model directly contributed to the observed reduction in non-operative time.

In a recent study, however, no difference in turnover time was found between employing a block room for upper extremity surgery and using $\mathrm{GA}^{20}$ suggesting that this variable is related to factors other than anesthetic technique, such as the time required for OR cleaning, equipment sterilization, and setup. Our model, on the other hand, uses a second form of parallel processing - two "swing" ORs which allows for OR cleaning and setup to occur while the preceding case is underway. When a dedicated block area and swing room model are used in combination, the gains in productivity from each process are compounded, which resulted in the marked decrease in non-operative time, or its overall elimination, and the increase in turnover and throughput. Of importance, we found that the swing room model resulted in similar productivity gains when we included patients who concomitantly received scheduled GA as the primary anesthetic technique in addition to BPB ( $n=28$ patients) (Figure 1; data not shown). In OR suites operating on the basis of the conventional "single surgeonsingle anesthesiologist-single OR" model, turnover in one OR may affect turnover in another OR, which has led to previous study of prolonged turnover times. ${ }^{21}$ Examples of non-anesthesia-controlled/non-surgical factors that affect non-operative time include delays due to OR cleaning as well as OR exit delays while waiting for a PACU slot. These inherently affect the "swing" room model to a significantly lesser extent, as larger time windows exist for the vacant swing ORs to be cleaned, and the high rate of PACU bypass eliminates a majority of potential delays owing to nursing

Table 2 Study outcome variables

\begin{tabular}{|c|c|c|c|c|c|}
\hline & Swing room & $n$ & Control & $n$ & $P$ value \\
\hline Non-operative time $(\mathrm{min})^{*}$ & $19[8-31]$ & 104 & $57[49-65]$ & 110 & $<0.0001$ \\
\hline Case throughput (number per 8-hr day) & $5.6[5.0-6.2]$ & 102 & $4.2[4.0-4.4]$ & 110 & $<0.0001$ \\
\hline Time from OR entry to incision (min) & $22[17-28]$ & 133 & $26[22-30]$ & 132 & $<0.0001$ \\
\hline Time from surgical closure to OR exit (min) & $6[4-8]$ & 131 & $7[5-10]$ & 133 & 0.001 \\
\hline PACU bypass & $80(60 \%)$ & 133 & $0(0 \%)$ & 133 & $<0.0001$ \\
\hline PACU length of stay $(\mathrm{min})^{\dagger}$ & $54[35-87]$ & 53 & 78 [65-117] & 133 & $<0.0001$ \\
\hline Postoperative opioid administration & $26(20 \%)$ & 133 & $109(82 \%)$ & 133 & $<0.0001$ \\
\hline Total opioid dose $(\mathrm{mg})^{*}$ & $4.6[4.0-10.0]$ & 26 & $12.0[7.3-20.0]$ & 109 & 0.0005 \\
\hline Rescue antiemetic administration & $3(2 \%)$ & 133 & $26(20 \%)$ & 133 & $<0.0001$ \\
\hline
\end{tabular}

Data are expressed as number $(\%)$ or median [interquartile range]. *Eleven percent of patients in the "swing room" group had negative nonoperative times. ${ }^{\dagger}$ Includes only patients unable to bypass PACU. ${ }^{*}$ Intravenous morphine equivalent dose administered per patient in those who received opioid analgesics. Sample sizes with $n<133$ were a result of i) missing chart data for study variables and ii) non-existing turnover times in patients who represented the first case of a day. OR $=$ operating room; PACU $=$ postanesthetic care unit 
and space shortages in the PACU. Our results from real-life abstracted data support this view. They are consistent with the computer simulation-based findings from a recent Finnish study comparing five different approaches to induction of anesthesia, which revealed that parallel processing models, regardless how they are structured, are more efficient than a traditionally sequenced care model. ${ }^{18}$

We point out that our present model, which was adapted to the main OR suite, produced a smaller increase in efficiency than the ambulatory setting in our previous study (33\% vs 56\%, respectively), as measured by estimated daily throughput ${ }^{13}$; however, this difference is to be seen in the context of the considerably longer surgical durations in the present study.

The use of our model also produced significantly improved patient recovery profiles. The high rate of PACU bypass in the swing room group is likely attributable to the decreased incidence of postoperative pain and PONV. In fact, fewer patients in the swing room group required rescue antiemetics, despite only $6 \%$ of patients in the swing room group $v s 71 \%$ of patients in the control group receiving PONV prophylaxis. It is likely that this is at least in part due to the markedly decreased requirement for postoperative opioids. These observations are of particular relevance when considered in the context of the large prospective study by Fortier et al., ${ }^{22}$ which found that postoperative pain and nausea are the leading causes of unanticipated hospital admission following surgery.

The swing room group bypassed the PACU in $60 \%$ of cases vs $0 \%$ of cases in the control group, and there was a significant reduction in the length of PACU stay in those unable to bypass the PACU. Although the present study was not designed to include an economic analysis, a high rate of PACU bypass and/or shorter PACU stay has the potential to decrease the burden of phase I recovery, which may lead to a lower peak PACU census, a variable that may be the primary determinant of PACU cost. ${ }^{23}$ Further studies regarding the economics of the swing room model are required.

Regarding human resources, the swing room model proved highly efficient from a surgical perspective. One single surgeon was able to perform an estimated $33 \%$ of additional cases with a mean duration of $70 \mathrm{~min}$ in an eight-hour day. Two anesthesiologists were required for this increase in throughput; however, the regional anesthesiologist was providing services to other ORs in addition to the swing ORs. In other words, the block room anesthesiologist and the anesthesia assistant who aids with block placement do not necessarily need to be exclusively dedicated to the swing room model. Four nurses are required for the two ORs in the swing room model, which corresponds to 1.4 cases per nurse (assuming a case load of
5.6 cases in an eight-hour day). At our centre, the conventional "single surgeon-single anesthesiologist-single OR model" employs 3.25 nurses per OR, which corresponds to 1.3 cases per nurse, assuming the completion of 4.2 cases in an eight-hour day.

The present study revealed a high block success rate, which was likely multifactorial, including the allowance of adequate time for the block to be effective and the use of ultrasound by a dedicated group of experienced regional anesthesiologists. Although ultrasound guidance, which allows direct visualization of local anesthetic spread around target nerves, has been shown to increase BPB success rates compared with traditional nerve localization techniques,${ }^{17}$ more research is required.

Our study is limited by its retrospective nature and the fact that its data are derived from a single academic tertiary care centre with experience in the implementation and usage of a swing room model. Therefore, our findings may not be easily reproducible at other centres. Also, because the daily slates of patients treated in the swing room model frequently included cases that did not meet inclusion criteria, we were unable to measure case throughput directly; rather, we had to estimate the number of comparable cases possible in an eight-hour day. However, our method represents a simple and straightforward tool in the post hoc analysis of throughput based on abstracted real-life data that can easily be modified to suit any period of interest.

In summary, in this case-matched cohort study in a typical mixed inpatient/outpatient population undergoing upper extremity orthopedic surgery, we found that the implementation of a "swing" room care model (based on ultrasound-guided regional anesthesia and parallel processing) in a hospital's main OR suite reduced nonoperative time, produced higher case throughput, and improved patient recovery profiles compared with the traditional "single surgeon-single anesthesiologist-single OR model". These results show that anesthesiology-driven innovation in the approach to the delivery of perioperative care represents a tool with the potential to contribute to a reduction of surgical wait lists in the public Canadian health care system.

Acknowledgements We sincerely thank Jeanette Kuper for her invaluable help with data abstraction. We also thank the anesthesia assistants at St. Paul's Hospital for their ongoing dedication to the regional anesthesia program. Finally, we are grateful to our orthopedic colleagues for their collaboration and support of the regional anesthesia swing room model.

Funding Department of Anesthesia, St. Paul's Hospital, Vancouver, B.C.; Department of Anesthesiology, Pharmacology \& Therapeutics, The University of British Columbia.

Conflict of interest None declared. 


\section{References}

1. Hadorn DC. Setting priorities for waiting lists: defining our terms. Steering Committee of the Western Canada Waiting List Project. CMAJ 2000; 163: 857-60.

2. Waddell JP. Improving waiting times for surgery. Can J Surg 2008; 51: 333-5.

3. Barua B, Rovere M. See you later! Wait times for access to health care in Canada remain unacceptably long. Fraser Forum. 2011 Jan/Feb; 18-20. Available from URL: http://www.fraserinstitute. org/uploadedFiles/fraser-ca/Content/research-news/research/articles/ see-you-later-wait-times.pdf (accessed July 2012).

4. Barua $B$. Why we wait: physician opinions on factors affecting health care wait times. Fraser Alert. 2011 Oct; 1-9. Available from URL: http://www.fraserinstitute.org/uploadedFiles/fraser$\mathrm{ca} /$ Content/research-news/research/publications/why-we-wait.pdf (accessed July 2012).

5. Chan VW, Peng PW, Kaszas Z, et al. A comparative study of general anesthesia, intravenous regional anesthesia, and axillary block for outpatient hand surgery: clinical outcome and cost analysis. Anesth Analg 2001; 93: 1181-4.

6. Hadzic A, Arliss J, Kerimoglu B, et al. A comparison of infraclavicular nerve block versus general anesthesia for hand and wrist day-case surgeries. Anesthesiology 2004; 101: 127-32.

7. McCartney CJ, Brull R, Chan VW, et al. Early but no long-term benefit of regional compared with general anesthesia for ambulatory hand surgery. Anesthesiology 2004; 101: 461-7.

8. Liu SS, Strodtbeck WM, Richman JM, Wu CL. A comparison of regional versus general anesthesia for ambulatory anesthesia: a meta-analysis of randomized controlled trials. Anesth Analg 2005; 101: 1634-42.

9. Hadzic A, Karaca PE, Hobeika P, et al. Peripheral nerve blocks result in superior recovery profile compared with general anesthesia in outpatient knee arthroscopy. Anesth Analg 2005; 100: 976-81.

10. Horn JL, Swide C, Gaebel BA, Cross RL Jr. Comparison of efficiency, recovery profile and perioperative costs of regional anaesthesia vs. general anaesthesia for outpatient upper extremity surgery. Eur J Anaesthesiol 2007; 24: 557-9.

11. O'Donnell BD, Ryan H, O'Sullivan O, Iohom G. Ultrasoundguided axillary brachial plexus block with 20 milliliters local anesthetic mixture versus general anesthesia for upper limb trauma surgery: an observer-blinded, prospective, randomized, controlled trial. Anesth Analg 2009; 109: 279-83.

12. Gonano C, Kettner SC, Ernstbrunner M, et al. Comparison of economical aspects of interscalene brachial plexus blockade and general anaesthesia for arthroscopic shoulder surgery. $\mathrm{Br} \mathrm{J}$ Anaesth 2009; 103: 428-33.

13. Head SJ, Seib R, Osborn JA, Schwarz SK. A "swing room" model based on regional anesthesia reduces turnover time and increases case throughput. Can J Anesth 2011; 58: 725-32.

14. Armstrong KP, Cherry RA. Brachial plexus anesthesia compared to general anesthesia when a block room is available. Can $\mathrm{J}$ Anesth 2004; 51: 41-4.

15. Williams $S R$, Chouinard $P$, Arcand $G$, et al. Ultrasound guidance speeds execution and improves the quality of supraclavicular block. Anesth Analg 2003; 97: 1518-23.

16. Soeding PE, Sha S, Royse CE, Marks P, Hoy G, Royse AG. A randomized trial of ultrasound-guided brachial plexus anaesthesia in upper limb surgery. Anaesth Intensive Care 2005; 33: 719-25.

17. Chan VW, Perlas A, McCartney CJ, Brull R, Xu D, Abbas S. Ultrasound guidance improves success rate of axillary brachial plexus block. Can J Anesth 2007; 54: 176-82.

18. Marjamaa RA, Torkki PM, Hirvensalo EJ, Kirvela OA. What is the best workflow for an operating room? A simulation study of five scenarios. Health Care Manag Sci 2009; 12: 142-6.

19. Krupka DC, Sathaye S, Sandberg WS. Reducing non-operative time: methods and impact on operating room economics. Int $\mathrm{J}$ Healthc Tech Manag 2008; 9: 325-52.

20. Mariano ER, Chu LF, Peinado CR, Mazzei WJ. Anesthesiacontrolled time and turnover time for ambulatory upper extremity surgery performed with regional versus general anesthesia. J Clin Anesth 2009; 21: 253-7.

21. Dexter F, Epstein RH, Marcon E, Ledolter J. Estimating the incidence of prolonged turnover times and delays by time of day. Anesthesiology 2005; 102: 1242-8.

22. Fortier J, Chung $F, S u J$. Unanticipated admission after ambulatory surgery-a prospective study. Can J Anaesth 1998; 45: 612-9.

23. Dexter F, Tinker JH. Analysis of strategies to decrease postanesthesia care unit costs. Anesthesiology 1995; 82: 94-101. 